\title{
Visual zapping in contemporary planning
}

Daniele Villa

Politecnico di Milano, Dipartimento di Architettura e Studi Urbani (daniele.villa@polimi.it)

Scevri da qualsiasi retorica passatista potrebbe essere illuminante ripensare per un istante alle modalità fisiche, tattili e analogiche di fruizione dei documenti che componevano gli strumenti urbanistici prima dell'era informatica. E in questo fugace salto all'indietro isolare i disegni, le mappe, le tavole, i lucidi, le eliocopie e il tempo stesso necessario per cercare, sfogliare, capire, confrontare. Le brevi considerazioni che seguono derivano da un'esperienza del tutto opposta, che è parte ordinaria del nostro quotidiano accesso potenzialmente illimitato a qualsiasi risorsa digitale. Può questa totale dicotomia aiutarci a dare alcune spiegazioni rispetto alle apparenti similitudini nei linguaggi visuali che caratterizzano l'urbanistica contemporanea, anche in contesti del tutto diversi? Ipotizzare una risposta affermativa significa per prima cosa tornare a confermare un assunto che sembra non dimostrare i suoi cinquant'anni suonati: il medium è il messaggio. E quando il medium è solo in apparenza sempre lo stesso (la standardizzata dotazione cartogragrafica del piano) ma il suo accesso e la sua fruizione sono del tutto mutati, dinamicizzati dal falsato tempo reale del browising web, le cose in parte si complicano. Più semplice iniziare concordando con il geografo Ola Söderström (Söderström, 1996) quando ci ricorda che, nonostante il planning sia un insieme di saperi e pratiche in cui la visualizzazione ha sempre giocato un ruolo cruciale, la mediazione delle immagini e la rappresentazione urbanistica rimangono ancora un territorio parzialmente inesplorato e a volte sottovalutato: «Consulting the theoretical works devoted to urban planning, however, one notices how little work has been done that analyses the relations between urban planning and the visual, or that, more particularly, takes seriously in its full complexity the question of the ways urban development and the modalities of representation of the city mutually structure each other» (Söderström, 1996: 250).

I carotaggi dentro alle tavole di piano delle ultime due copertine di Territorio (numeri 94 e 95) sono tasselli iniziali di un'operazione che gioca volutamente e forzosamente sulla superficie del visuale con un'intenzione definita: scorrere e affiancare immagini di luoghi, politiche, intenzioni, azioni progettuali diverse e per lo più non comparabili, per trarne un fermo immagine tanto parziale quanto forse capace di restituirci alcune dominanti, segni comuni di un modo di disegnare l'urbanistica dell'ultimo decennio. Questo esperimento in vitro, tornando alle considerazioni iniziali, è reso possibile e al contempo distorto dal modo stesso di accedere, collezionare, scorrere centinaia di tavole nel tempo condensato del web e nello spazio racchiuso e intangibile del monitor.

Due gli elementi che accomunano questo zapping urbanistico: lo sguardo a una scala che non vada molto oltre l'urbano e la selezione di tavole di impianto generale, per lo più sintetiche $o$ strategiche. La principale ragione di questa scelta è data dall'attendibile uniformità che accomuna gran parte degli strumenti visuali con finalità quantitativo-prescrittiva (Dühr, 2006). Salta immediatamente all'occhio il ben diverso dispiegamento dei codici grafici più disparati nelle tavole-manifesto, nei poster-plan, nelle dotazioni iconografiche delle relazioni di piano che sfuggono all'ortodossia planimetrica in una ibridazione a volte parossistica di idioletti grafici. Questa molteplicità di approcci alla codificazione visiva apre inevitabilmente a una domanda sulla funzionalità, latente o manifesta, di queste rappresentazioni urbanistiche accomunate da un eccesso visivo che potremmo definire rumoroso. Secondo Alberto Cairo (Cairo, 2013) la funzionalità comunicativo-grafica dovrebbe restringere il campo e la varietà delle possibili espressioni visuali eppure, in alcuni degli esempi proposti, sembrerebbe prevalere una assenza di progettualità comunicativa, un dubbio di fondo innanzitutto sui soggetti a cui gli artefatti sono destinati. Come se, paradossalmente, le grandi visioni strategiche rimanessero opache, mentre le prescrizioni dei documenti regolatori riuscissero a mantenere rigore, chiarezza e robustezza visiva. Una rappresentazione tassonomica ormai 'stabilizzata' (Drucker, 1992: 23), in parte figlia di quello che Söderström chiama visual order of the civil servant facendola risalire al primo best seller fra i manuali urbanistici: il lavoro di Reinhard Baumeister.

«The urban planning of the civil servants, so called because it was the product of men for the most part employed in municipal administration formulating laws, rules, and manuals, nevertheless played a foundational role in the codifying of urban planning in many countries. This influence was not, of course, felt everywhere immediately. In most cases one has to wait until the 1920s and 1930s, when the evident drawbacks of laissez faire economics forced countries to develop their urban planning systems» (Söderström, 1996: 262). È anche il supporto cartografico, la mappacarta (Gabellini, 1996), con la sua immediatezza interpretativa, a conferire una apparente naturale assertiva oggettività ai disegni regolatori del piano, mentre lo spazio comunicativo dei documenti strategici si deve confrontare con un sovrapporsi di stratificazioni di senso non sempre organiche, spesso ibridate da culture visuali molto diverse e non facilmente congruenti. 


\begin{tabular}{|l|}
\hline \\
\hline Messina, 2018, Piano Regolatore Generale \\
\hline Siena, 2007, Piano Strutturale \\
\hline Milano, 2019, Piano di Governo del Territorio \\
\hline Ferrara, 2008, Piano Strutturale Comunale \\
\hline
\end{tabular}

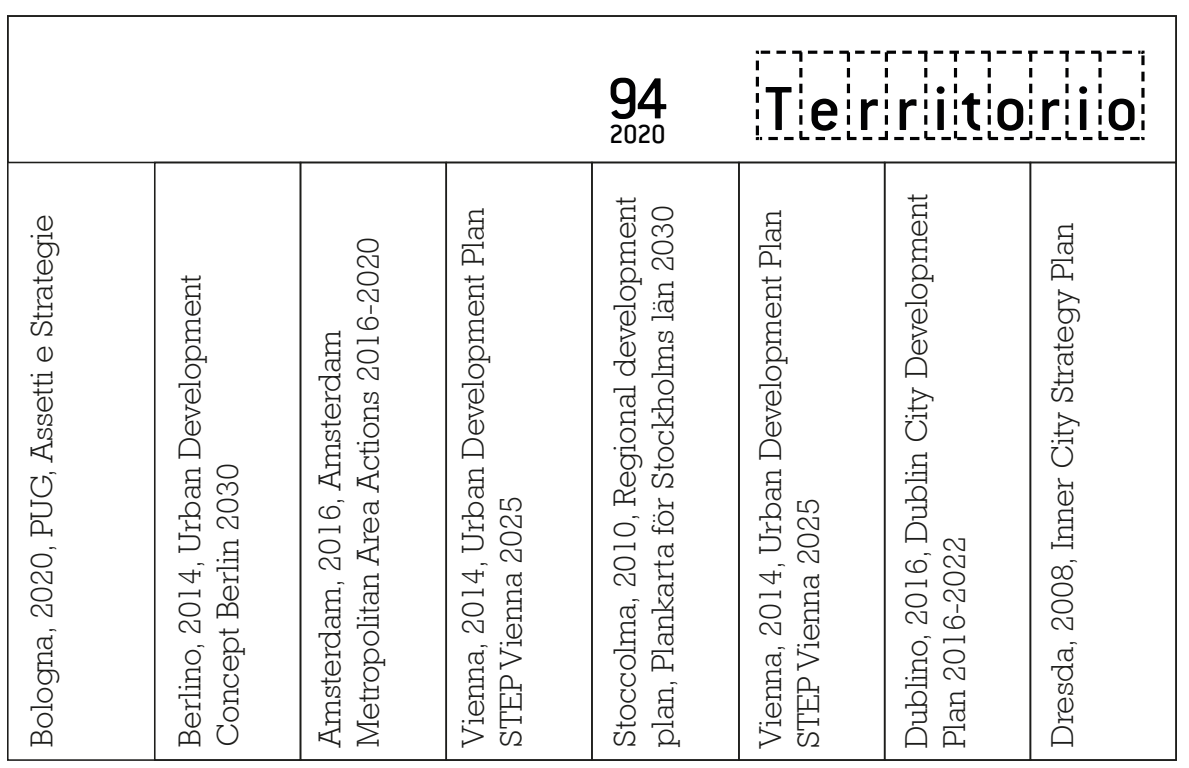

Guardando a questa dualità a cavallo fra il rigore storicizzato dello zoning e il melting pot plurilinguistico delle tavole-manifesto torna ad avere senso domandarsi quale siano gli obiettivi di buon funzionamento dell'immagine urbanistica e infine come possiamo tornare a ridefinire cosa sia per noi, oggi, l'efficacia della rappresentazione nel planning. Ola Söderström ci viene in aiuto con una valida classificazione di efficacia interna, riferita alla necessità di semplificare utilmente la complessità del reale per rendere comprensibili visivamente solo alcune sue caratteristiche, ed efficacia esterna relativa alle potenzialità persuasive del linguaggio visivo, che deve essere in grado far cogliere a un pubblico potenzialmente ampio le relazioni complesse fra le identità dei luoghi e la spazializzazione delle scelte future. In questo il sapere esperto deve agire un costante bilanciamento fra le procedure di codificazione tecnica, spesso iper-formalizzate, e le necessità di un racconto visivo allargato a una percezione sociale plurale. Non possiamo però cadere nel tranello della retorica generalista: gli strumenti di pianificazione urbana hanno l'intrinseca necessità di rivolgersi a lettori diversi, con registri adatti alla natura a volte estremamente settoriale di alcuni dei dispositivi necessari alla messa in atto delle scelte.

L'osservazione velocizzata da cui derivano i dodici 'vetrini' che compongono le ultime due copertine di Territorio si concentra però sul livello comunicativo solo apparentemente più di superficie: quello diretto alla divulgazione e pubblicizzazione delle strategie dei piani. Su questo crinale non del tutto distinto fra il rigore della tavola tecnica e la presunta seduttività del manifesto pubblicitario sembrano condensarsi gran parte delle difficoltà di messa a fuoco di un lessico grafico icastico.

Una prima ragione può rintracciarsi nella necessità di uscire dai propri steccati tecnico disciplinari da parte del saper fare 
urbanistico, riconoscendo l'opportunità di acquisire competenze prettamente comunicative, sempre più specialistiche, e connotate da un necessario opportunismo epistemologico: «Ma il comunicatore visivo è condannato a praticare quella regola che il grande Einstein chiamava opportunismo epistemologico. Cioè lavorare di referenza, di percezione, di qualità sensoriale ed estetica. Il progetto è condannato alla mutidisciplinarità: anche quando non lo sa, è globale, è sempre design totale» (Anceschi, 1992: 7). Una seconda questione è in parte connessa ai fattori dominanti della cultura visuale-digitale contemporanea nel suo essere prepotentemente bulimica, disomogenea, caotica, ipertrofica, rumorosa. È proprio il paragone acustico ad apparire appropriato: è sempre più difficile limitare il ricorso a stilemi visuali rumorosi e, per questo, incapaci di permettere una lettura lineare delle relazioni interne agli artefatti comunicativi. Il rumore grafico, facendo prevalere il dominio percettivo di elementi poco calibrati, depotenzia ineluttabilmente una delle grandi conquiste dell'informatizzazione: la possibilità di gestire e rendere leggibili livelli sovrapposti di segni, dosando pesi e trasparenze.

Non si tratta di avventurarsi in considerazioni estetiche o di gusto, piuttosto è necessario prendere atto della necessità di acuire le competenze di visual thinking proprio dove un'apparente uniformità semantica è messa in discussione dal trasformarsi dei mezzi di accumulazione, diffusione e manipolazione delle immagini.

La rappresentazione urbanistica ha costruito e storicizzato una compattezza tassonomica, diagrammatica e cartografica (Massironi, 1982) basata su un assunto di fondo: i soggetti produttori e fruitori dei suoi apparati visuali appartengono per lo più al campo circoscritto dei tecnici e dei decisori, ma le cose nel frattempo sono profondamente cambiate e la crescente domanda sociale di inclusione e comunicazione ha innescato un inevitabile ripensamento del ruolo divulgativo di molti dei materiali illustrativi del piano. Sotto questa spinta il rischio di una progettazione visuale parziale e non attenta tende a generare disegni di territorio che oscillano da un'iconicità laconica, stilizzata e astratta all'opposta figuratività ridondante, dove ogni segno sembra voler didascalicamente dichiarare la propria funzione. Fra le manipolazioni necessarie per produrre un materiale grafico funzionale (selezione, enfatizzazione, esclusione, sovrapposizione, comparazione, ecc.) l'eccesso auto-didascalico rappresenta sicuramente uno dei tratti del nostro tempo e uno dei fattori, assieme al rumore grafico, di evidente riduzione dell'efficacia generale di una rappresentazione territoriale complessa. L'immediatezza della comunicazione non è infatti pretesa velleitaria di annullamento dello sforzo cognitivo da parte del fruitore, ma attenta ricucitura dei nessi di senso necessari alla piena comprensione: «Un testo verbale necessario a definire la cosa rappresentata e i legami intercorrenti fra i segni grafici utilizzati e i contenuti di pensiero da essi vincolati. Quest'oggetto prenderà la forma di legenda, di didascalia o di argomentazione teorica, mentre i tratti grafici saranno costruiti in funzione di una intrinseca analogia con i pensieri espressi» (Massironi, 1982: 138). Lasciare alle didascalie il loro spazio significa anche ricordare quanto il processo di rappresentazione urbanistica non si produca ex post, a valle di analisi e scelte, ma sia esso stesso un pezzo della fase euristica, di scoperta e ideazione che, proprio per questo, necessita di un'azione di decodificazione non sempre auto-evidente.

Un'ultima considerazione riguarda un principio o meglio un effetto che, parafrasando Wim Wenders (Wenders, 1993), potremmo definire controdisegno. Wenders, riferendosi alla fotografia, la descrive come un atto bidirezionale, che produce sempre una traccia latente di chi ha fotografato e al contempo lascia nel fotografo un segno di quello scatto, una sorta di controscatto. Parallelamente il controdisegno potrebbe essere inteso come quello che resta all'autore dopo che la rappresentazione è stata progettata e realizzata, sia rispetto all'arricchimento del proprio lessico visuale, sia in termini di affinamento della propria capacità di costruire artefatti comunicativi più efficaci. Lo zapping fra centinaia di visualizzazioni di figure di piano restituisce la sensazione di un impoverimento generalizzato del controdisegno come se, per certi versi, il mare magnum dei riferimenti visivi d'origine tendesse ad assomigliarsi e, d'altro canto, la modalità di fruizione accelerata operasse un'ulteriore spinta alla superficialità e alla non sedimentazione dell'esperienza. La molteplicità dei saperi che necessariamente ruotano attorno al disegno urbanistico contemporaneo non devono farci distogliere lo sguardo dalla necessità di ripensare, operativamente, le caratteristiche di una comunicazione visuale efficace e capace di confrontarsi con necessità specialistiche e insieme divulgative, non scindibili dalla tecnologia con cui le immagini vengono prodotte e fruite. Ciò non significa accondiscendere a una retorica grafica stereotipata utilizzando acriticamente risorse e strumenti di cui è necessario continuare a ribadire la non-neutralità.

\section{Riferimenti bibliografici}

Anceschi G., 1992, L’oggetto della raffigurazione. Milano: ETAS.

Cairo A., 2013, L'arte funzionale. Infografica e visualizzazione delle informazioni. Pearson: Milano.

Drucker J., 1992, Graphesis-Visual Forms of Knowledge Production. Cambridge: Harvard University Press.

Dühr S., 2006, The Visual Language of Spatial Planning: Exploring Cartographic Representations for Spatial Planning in Europe. London: Routledge.

Gabellini P., 1996, Il disegno urbanistico. Roma: Carocci.

Söderström O., 1996, «Paper City: Visual Thinking in Urban Planning». Ecumene, 3: 249-281.

Massironi M., 1982, Vedere con il disegno. Padova: Franco Muzzio. Wenders W., 1993, Una Volta. Roma: Socrates. 